\title{
The average binding number of graphs
}

\author{
Ersin Aslan \\ Department of Software Engineering, Celal Bayar University, Turgutlu-Manisa, Turkey
}

e-mail: ersin.aslan@cbu.edu.tr

Received 29 Sep 2018

Accepted 3 Jan 2019

\begin{abstract}
The binding number is a measure of the vulnerability of a graph. We investigate a refinement that involves the average of this parameter. Like the binding number itself, the average binding number bind ${ }_{\text {av }}(G)$ of $G$ measures the vulnerability of a graph, which is more sensitive. In this study, some bounds of the average binding number of some special graphs are obtained. Furthermore some results about the average binding number of graphs obtained from graph operations are also provided.
\end{abstract}

KEYWORDS: graph vulnerability, network design and communication, connectivity, average lower connectivity

MSC2010: 05C40 68M10 68R10

\section{INTRODUCTION}

One of the most important problems which is solved by the help of graph theory is to design a network model whose resistance for the disruptions is more than other networks. Graphs are often used to model real world problems such as in a communication, computer, or spy network. In a network, the vulnerability parameters measure the resistance of the network to disruption of operation after the failure of certain stations or links. Parameters used to measure the vulnerability of networks include connectivity ${ }^{4}$ average lower connectivity ${ }^{1}$, binding number ${ }^{13}$, etc.

Let $G$ be a finite simple graph with vertex set $V(G)$ and edge set $E(G)$. The neighbourhood of a vertex $u \in V(G)$ is the set $N(u):=\{v \in V(G) \mid$ $u v \in E(G)\}$. This is more commonly called the open neighbourhood of a vertex. The neighbourhood of a set $S \subseteq V(G)$ is $N(S)=\cup_{u \in S} N(u)$. Let $d(u)$ denote the degree of the vertex $u$ in $G$. The maximum degree of a graph $G$ is the largest vertex degree of $G$, denoted $\Delta(G)$, and similarly, the minimum vertex degree is the smallest vertex degree of $G$, denoted $\delta(G)$. The distance $d(u, v)$ between two vertices $u$ and $v$ of a finite graph is the minimum length of the paths connecting them, i.e., the length of a graph geodesic. The diameter $d$ of a graph is the greatest distance between any pair of vertices. A minimum vertex cover is a vertex cover having the smallest possible number of vertices for a given graph. The size of a minimum vertex cover of a graph $G$ is known as the vertex cover number and is denoted
$\alpha(G)$. An independent vertex set of a graph $G$ is a subset of the vertices such that no two vertices in the subset represent an edge of $G$. Given a vertex cover of a graph, all vertices not in the cover define a independent vertex set. A maximum independent vertex set is an independent vertex set containing the largest possible number of vertices for a given graph. The independence number of $G$, denoted by $\beta(G)$, is the number of vertices in a maximum independent set of $G$. A spanning subgraph of $G$ is a subgraph that contains every vertex of $G$.

Aslan ${ }^{1}$ introduced the concept of average lower connectivity. For a vertex $v$ of a graph $G$, the lower connectivity at $v$, denoted $s_{v}(G)$, is the smallest number of vertices in a set that contains $v$ whose deletion from $G$ produces a disconnected or a trivial graph. The average lower connectivity denoted by $\kappa_{\mathrm{av}}(G)$, is the value $\Sigma_{v \in V(G)} s_{v}(G) / n$, where $n$ denotes the number of vertices in the graph $G$ and $\Sigma_{v \in V(G)} S_{v}(G)$ denotes the sum over all vertices of $G$.

Woodall $^{13}$ defined the binding number of a graph $G$ as

$$
\operatorname{bind}(G)=\min _{S \in F(G)}\left\{\frac{|N(S)|}{|S|}\right\}
$$

where $F(G)=\{S \subseteq V(G) \mid S \neq \varnothing, N(S) \neq V(G)\}$. A binding set of $G$ is any set $S$ such that $\operatorname{bind}(G)=$ $|N(S)| /|S|$.

The study of binding number in graphs is an important research area, perhaps also the fastestgrowing area within graph theory. The reason for the steady and rapid growth of this area may be the diversity of its applications to both theoretical 


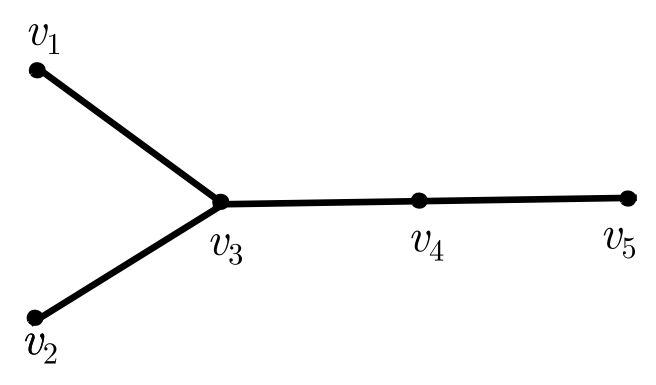

Fig. 1 The graph $G$.

and real world problems. More recently interest has been rearoused, yielding a succession of results covering inter alia product graphs $\mathrm{s}^{7,11,12}$, cliques and cycles as subgraphs, and the range of possible values of binding numbers ${ }^{8}$. Linzhung et $\mathrm{al}^{9}$ have extended the binding number to the edges and the studied the edge-binding number of some plane graph.

The average parameters have been found to be more useful in some circumstances than the corresponding measures based on worst-case situations $s^{2,3,6,10}$. Thus incorporating the concept of the binding number and the idea of the average lower connectivity introduces a new graph parameter called the average binding number, $\operatorname{bind}_{\mathrm{av}}(G)$.

For $v \in V(G)$, the local binding number of $v$ is

$$
\operatorname{bind}_{v}(G)=\min _{S \in F_{v}(G)}\left\{\frac{|N(S)|}{|S|}\right\},
$$

where $F_{v}(G)=\{S \subseteq V(G) \mid v \in S, S \neq \varnothing, N(S) \neq$ $V(G)\}$. Clearly,

$$
\operatorname{bind}(G)=\min _{v \in V(G)}\left\{\operatorname{bind}_{v}(G)\right\} .
$$

A local binding set of $v$ in $G$ is $S \in F_{v}(G)$ such that $\operatorname{bind}_{v}(G)=|N(S)| /|S|$. Furthermore, the average binding number of $G$ is defined as

$$
\operatorname{bind}_{\mathrm{av}}(G)=\frac{1}{n} \sum_{v \in V(G)} \operatorname{bind}_{v}(G),
$$

where $n$ is the number of vertices in graph $G$.

Example 1 Consider the graph $G$ in Fig. 1, where $|V(G)|=5$ and $|E(G)|=4$. Note that bind $_{v_{1}}=\frac{1}{2}$, $\operatorname{bind}_{v_{2}}=\frac{1}{2}, \operatorname{bind}_{v_{3}}=\frac{4}{4}=1, \operatorname{bind}_{v_{4}}=\frac{2}{3}$, and bind $v_{5}=$ $\frac{2}{3}$. It follows that

$$
\operatorname{bind}_{\mathrm{av}}(G)=\frac{1}{5}\left(\frac{1}{2}+\frac{1}{2}+1+\frac{2}{3}+\frac{2}{3}\right)=\frac{2}{3} .
$$

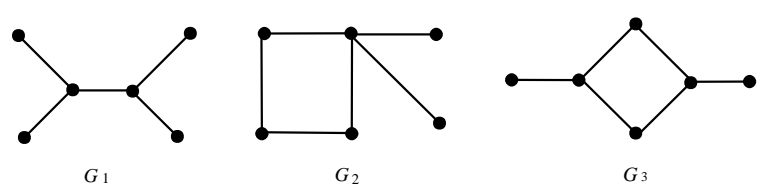

Fig. 2 The graphs $G_{1}, G_{2}$ and $G_{3}$.
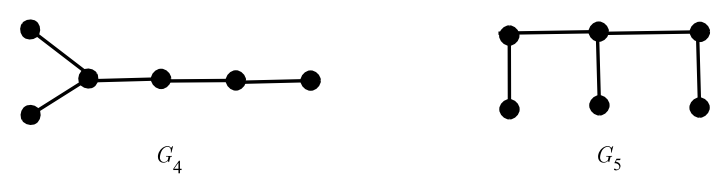

Fig. 3 The graphs $G_{4}$ and $G_{5}$.

The following examples show that the average binding number is more efficient than the connectivity, the average lower connectivity, and the binding number in measuring the vulnerability of some graphs.

Example 2 It is easy to see that connectivity of a star $K_{1,4}$ and a path $P_{5}$ are equal,

$$
\kappa\left(K_{1,4}\right)=\kappa\left(P_{5}\right)=1 \text {. }
$$

however, the average binding number of a star $K_{1,4}$ and a path $P_{5}$ are different,

$$
\operatorname{bind}_{\mathrm{av}}\left(K_{1,4}\right)=1, \quad \operatorname{bind}_{\mathrm{av}}\left(P_{5}\right)=\frac{14}{15} .
$$

Example 3 Let $G_{1}, G_{2}$, and $G_{3}$ be the graphs in Fig. 2. It is easy to see that the connectivity and binding number of $G_{1}, G_{2}$, and $G_{3}$ are equal;

$$
\begin{array}{r}
\kappa\left(G_{1}\right)=\kappa\left(G_{2}\right)=\kappa\left(G_{3}\right)=1, \\
\operatorname{bind}\left(G_{1}\right)=\operatorname{bind}\left(G_{2}\right)=\operatorname{bind}\left(G_{3}\right)=\frac{1}{2} .
\end{array}
$$

However, the average binding number of $G_{1}, G_{2}$, and $G_{3}$ are different,

$\operatorname{bind}_{\mathrm{av}}\left(G_{1}\right)=\frac{3}{5}, \operatorname{bind}_{\mathrm{av}}\left(G_{2}\right)=\frac{81}{120}, \operatorname{bind}_{\mathrm{av}}\left(G_{3}\right)=\frac{2}{3}$.

Example 4 Let $G_{4}$ and $G_{5}$ be the graphs in Fig. 3. It is easy to check that the average lower connectivity of $G_{4}$ and $G_{5}$ are equal,

$$
\kappa_{\mathrm{av}}\left(G_{4}\right)=\kappa_{\mathrm{av}}\left(G_{5}\right)=\frac{3}{2} .
$$

However, the average binding number of $G_{1}$ and $G_{2}$ are different,

$$
\operatorname{bind}_{\mathrm{av}}\left(G_{4}\right)=\frac{2}{3}, \quad \operatorname{bind}_{\mathrm{av}}\left(G_{5}\right)=1 .
$$




\section{BOUNDS FOR AVERAGE BINDING NUMBER}

In this study, some bounds of the average binding number are obtained for some special graphs. Furthermore some results of the average binding number of graphs generated by graph operations are also given. The related theorems of the average binding number and other graph parameters are provided as the followings.

Theorem 1 If $G$ is a graph of order $n$ with the minimum degree $\delta(G)$, then

$$
\operatorname{bind}_{\mathrm{av}}(G) \geqslant \frac{\delta(G)}{n-\delta(G)} .
$$

Proof: Let $v \in V(G)$ and $S_{v}$ be a local binding set at $v$. Certainly $\left|N\left(S_{v}\right)\right| \geqslant d(v) \geqslant \delta(G)$. Since $N\left(S_{v}\right) \neq$ $V(G), S_{v}$ omit all the neighbours of some vertex $w$, and $\left|S_{v}\right| \leqslant n-d(w) \leqslant n-\delta(G)$. Thus

$$
\operatorname{bind}_{v}(G)=\frac{\left|N\left(S_{v}\right)\right|}{\left|S_{v}\right|} \geqslant \frac{\delta(G)}{n-\delta(G)} .
$$

Hence

$$
\operatorname{bind}_{\mathrm{av}}(G)=\frac{1}{n} \sum_{v \in V(G)} \operatorname{bind}_{v}(G) \geqslant \frac{\delta(G)}{n-\delta(G)} .
$$

Theorem 2 If $G$ is a graph of order $n$ with covering number $\alpha(G)$ and independence number $\beta(G)$, then

$$
\operatorname{bind}_{\mathrm{av}}(G) \leqslant \frac{\alpha(G)(\beta(G)+n-1)}{n \beta(G)} .
$$

Proof: Let $v \in V(G)$ and $M$ be a maximum independent set of $G$.

If $v \in V(M)$, then there is a local binding set $S_{v} \in F_{v}(G)$ that contains all vertices in the maximum independent set of $G$, and $\left|S_{v}\right|=\beta(G)$. Then $\left|N\left(S_{v}\right)\right|=\alpha(G)$ and $\operatorname{bind}_{v}(G)=\alpha(G) / \beta(G)$.

If $v \notin V(M)$, then for $S \in F_{v}(G),|S| \geqslant \beta(G)$ and $|N(S)| \leqslant n-1$. Thus $\operatorname{bind}_{v}(G) \leqslant(n-1) / \beta(G)$. Hence

$$
\begin{aligned}
\operatorname{bind}_{\mathrm{av}}(G) & \leqslant \frac{\beta(G)\left(\frac{\alpha(G)}{\beta(G)}\right)+\alpha(G)\left(\frac{n-1}{\beta(G)}\right)}{n} \\
& =\frac{\alpha(G)(\beta(G)+n-1)}{n \beta(G)} .
\end{aligned}
$$

Theorem 3 If $G$ is a graph of order $n$ with the minimum degree $\delta(G)$ and maximum degree $\Delta(G)$, then

$$
\operatorname{bind}_{\mathrm{av}}(G) \geqslant \frac{\delta(G)}{\Delta(G)+1} .
$$

Proof: Let $v \in V(G)$. For $S_{v} \in F_{v}(G),\left|S_{v}\right| \leqslant \Delta(G)+1$ and $\left|N\left(S_{v}\right)\right| \geqslant \delta(G)$, hence

$$
\operatorname{bind}_{v}(G)=\min _{S_{v} \in F_{v}(G)} \frac{\left|N\left(S_{v}\right)\right|}{\left|S_{v}\right|} \geqslant \frac{\delta(G)}{\Delta(G)+1} .
$$

Thus

$$
\operatorname{bind}_{\mathrm{av}}(G)=\frac{1}{n} \sum_{v \in V(G)} \operatorname{bind}_{v}(G) \geqslant \frac{\delta(G)}{\Delta(G)+1} .
$$

Theorem 3 implies that $\operatorname{bind}_{\mathrm{av}}(T) \geqslant 1 /(\Delta(T)+1)$ for a tree $T$.

Theorem 4 If $H$ is a spanning subgraph of $G$, then

$$
\operatorname{bind}_{\mathrm{av}}(H) \leqslant \operatorname{bind}_{\mathrm{av}}(G) .
$$

Proof: Let $v \in V(G) \cap V(H)$ with a local binding set $S_{v}^{*} \in F_{v}(G)$ of $G$. Let denote $N_{G}\left(S_{v}^{*}\right)=N\left(S_{v}^{*}\right) \cap V(G)$ and $N_{H}\left(S_{v}^{*}\right)=N\left(S_{v}^{*}\right) \cap V(H)$. Then $N_{H}\left(S_{v}^{*}\right) \subseteq N_{G}\left(S_{v}^{*}\right)$ and

$$
\operatorname{bind}_{v}(H) \leqslant \frac{\left|N_{H}\left(S_{v}^{*}\right)\right|}{\left|S_{v}^{*}\right|} \leqslant \frac{\left|N_{G}\left(S_{v}^{*}\right)\right|}{\left|S_{v}^{*}\right|}=\operatorname{bind}_{v}(G) .
$$

Thus by the definition, $\operatorname{bind}_{\mathrm{av}}(H) \leqslant \operatorname{bind}_{\mathrm{av}}(G)$.

Theorem 5 If $G$ is a graph of order $n$, then

$$
\operatorname{bind}(G) \leqslant \operatorname{bind}_{\mathrm{av}}(G) .
$$

Proof: From the definitions it is clear that for $v \in$ $V(G)$, $\operatorname{bind}(G) \leqslant \operatorname{bind}_{v}(G)$. Thus

$$
\operatorname{bind}(G) \leqslant \frac{1}{n} \sum_{v \in V(G)} \operatorname{bind}_{v}(G)=\operatorname{bind}_{\mathrm{av}}(G) .
$$

\section{AVERAGE BINDING NUMBER OF CLASSES OF GRAPHS}

Theorem 6 If $P_{n}$ is a path of order $n \geqslant 3$, then

$$
\operatorname{bind}_{\mathrm{av}}\left(P_{n}\right)= \begin{cases}1, & n \text { even } \\ \frac{2 n^{2}-5 n+3}{2 n^{2}-4 n}, & n \text { odd }\end{cases}
$$

Proof: Let $v \in V\left(P_{n}\right)$ and $S_{v} \in F_{v}\left(P_{n}\right)$.

Case 1: $n$ is even. Since $\left|N\left(S_{v}\right)\right| \geqslant\left|S_{v}\right|$ with the equality holds when $S_{v}$ is a maximum independent set of $P_{n}$, therefore, $\operatorname{bind}_{v}\left(P_{n}\right)=1$ for all $v$, which implies $\operatorname{bind}_{\mathrm{av}}\left(P_{n}\right)=1$.

Case 2: $n$ is odd. Let the vertices of $P_{n}$ be $p_{1}, p_{2}, \ldots, p_{n}$ in order along the path. There is a 
maximum independent set that gives the unique independence number $\beta\left(P_{n}\right)=\frac{1}{2}(n+1)$.

For any $v$ in the maximum independent set containing $\frac{1}{2}(n+1)$ vertices, the $\operatorname{bind}_{v}\left(P_{n}\right)$ is obtained when $\left|S_{v}\right|=\beta\left(P_{n}\right)=\frac{1}{2}(n+1)$ and $\left|N\left(S_{v}\right)\right|=\frac{1}{2}(n-1)$.

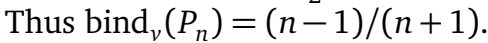

For any $v$ not in a maximum independent set of $\frac{1}{2}(n-1)$ vertices, to obtain $\operatorname{bind}_{v}\left(P_{n}\right)$ we require $S_{v}$ as large as possible without $N\left(S_{v}\right)$ being the whole of $V\left(P_{n}\right)$, i.e., $\left|S_{v}\right|=n-2$, for example, $S_{v}=$ $\left.\left\{p_{3}, p_{4}, \ldots, p_{n}\right\}\right)$. Thus bind $\left(P_{n}\right)=(n-1) /(n-2)$.

Hence, by the definition

$$
\begin{aligned}
\operatorname{bind}_{\mathrm{av}}\left(P_{n}\right) & =\frac{1}{n}\left(\frac{(n+1)(n-1)}{2(n+1)}+\frac{(n-1)(n-1)}{2(n-2)}\right) \\
& =\frac{2 n^{2}-5 n+3}{2 n^{2}-4 n} .
\end{aligned}
$$

Theorem 7 If $C_{n}$ is a cycle of order $n \geqslant 4$, then

$$
\operatorname{bind}_{\mathrm{av}}\left(C_{n}\right)= \begin{cases}1, & n \text { even, } \\ \frac{n-1}{n-2}, & n \text { odd. }\end{cases}
$$

Proof: Let $v \in V\left(C_{n}\right)$ and $S_{v} \in F_{v}\left(C_{n}\right)$.

Case 1: $n$ is even. For all $v \in V\left(C_{n}\right)$, if $\left|S_{v}\right|=r$ then $\left|N\left(S_{v}\right)\right| \geqslant r$, therefore, $\operatorname{bind}_{v}\left(C_{n}\right) \geqslant 1$. Since there is a local binding set $S_{v}^{*}$ of $C_{n}$ such that $\left|S_{v}^{*}\right|=$ $\frac{1}{2} n$, when $S_{v}^{*}$ is a maximum independent set of $C_{n}$, and $\left|N\left(S_{v}^{*}\right)\right|=\frac{1}{2} n$. Hence $\operatorname{bind}_{v}\left(C_{n}\right)=1$, for $v \in$ $V\left(C_{n}\right)$, and therefore, $\operatorname{bind}_{\mathrm{av}}\left(C_{n}\right)=1$

Case 2: $n$ is odd. For $\left|S_{v}\right|=r$, then $r \leqslant$ $n-\delta\left(C_{n}\right)=n-2$ and $\left|N\left(S_{v}\right)\right| \geqslant r+1$. Thus $\left|N\left(S_{v}\right)\right| /\left|S_{v}\right| \geqslant(r+1) / r$. is a decreasing function of $r$ that has the minimum value when $r=n-2$. Hence $\operatorname{bind}_{v}\left(C_{n}\right) \geqslant(n-1) /(n-2)$. Since there is a binding set $S_{v}^{*}$ of $C_{n}$ such that $\left|S_{v}^{*}\right|=n-2$ and $\left|N\left(S_{v}\right)\right|=n-1$, thus $\operatorname{bind}_{v}\left(C_{n}\right)=(n-1) /(n-2)$, and therefore, $\operatorname{bind}_{\mathrm{av}}\left(C_{n}\right)=(n-1) /(n-2)$.

Theorem 8 If $K_{n}$ is a complete graph of order $n \geqslant 2$, then $\operatorname{bind}_{\mathrm{av}}\left(K_{n}\right)=n-1$.

Proof: Let $v \in V\left(K_{n}\right)$ and $S_{v} \in F_{v}\left(K_{n}\right)$. If $\left|S_{v}\right| \geqslant 2$, then $N\left(S_{v}\right)=V\left(K_{n}\right)$, which contradicts with $S_{v} \in$ $F_{v}\left(K_{n}\right)$. Thus $\left|S_{v}\right|=1$ and $\left|N\left(S_{v}\right)\right|=n-1$. Hence $\operatorname{bind}_{v}\left(K_{n}\right)=n-1$ for all $v \in V\left(K_{n}\right)$ and $\operatorname{bind}_{\text {av }}\left(K_{n}\right)=$ $n-1$.

Theorem 9 If $K_{a, b}$ is a complete bipartite graph of order $a+b$ with $1 \leqslant a \leqslant b$, then

$$
\operatorname{bind}_{\mathrm{av}}\left(K_{a, b}\right)=1 .
$$

Proof: Let $V\left(K_{a, b}\right)=V\left(G_{1}\right) \cup V\left(G_{2}\right)$ be the vertex set of $K_{a, b}$, where the set $V\left(G_{1}\right)$ contains $a$ vertices having degree $b$ and the set $V\left(G_{2}\right)$ contains $b$ vertices having degree $a$. For $v \in V\left(K_{a, b}\right)$ and $S_{v} \in$ $F_{v}\left(V\left(K_{a, b}\right)\right)$, if $\left|S_{v} \cap V\left(G_{1}\right)\right| \neq 0$ and $\left|S_{v} \cap V\left(G_{2}\right)\right| \neq 0$ then $N\left(S_{v}\right)=V\left(K_{a, b}\right)$, a contradiction. This implies that $S_{v} \subseteq V\left(G_{1}\right)$ to have $N\left(S_{v}\right)=V\left(G_{2}\right)$, or $S_{v} \subseteq V\left(G_{2}\right)$ to have $N\left(S_{v}\right)=V\left(G_{1}\right)$. Since for $a$ vertices of $v \in V\left(G_{1}\right),\left|S_{v}\right| \leqslant a$ and $\left|N\left(S_{v}\right)\right|=b$, i.e., $\operatorname{bind}_{v}\left(K_{a, b}\right)=b / a$, and for $b$ vertices of $v \in V\left(G_{2}\right)$, $\left|S_{v}\right| \leqslant b$ and $\left|N\left(S_{v}\right)\right|=a$, i.e., $\operatorname{bind}_{v}\left(K_{a, b}\right)=a / b$. Therefore,

$$
\operatorname{bind}_{\mathrm{av}}\left(K_{a, b}\right)=\frac{1}{a+b}\left(a \frac{b}{a}+b \frac{a}{b}\right)=1 .
$$

Corollary 1 If $K_{1, n}$ is a star graph, then

$$
\operatorname{bind}_{\mathrm{av}}\left(K_{1, n}\right)=1 .
$$

\section{GRAPH OPERATIONS}

This section provides some results of the average binding number of graphs obtained from graph operators.

\section{Power of a graph}

Definition 1 [Ref. 5] The $k$ th power, $G^{k}$, of a connected graph $G$ is the graph with $V\left(G^{k}\right)=V(G)$ for which $u v \in E\left(G^{k}\right)$ if $1 \leqslant d(u, v) \leqslant k$.

Theorem 10 If $G$ is a graph of order $n$ and diameter $d$, then

$\operatorname{bind}_{\mathrm{av}}(G) \leqslant \operatorname{bind}_{\mathrm{av}}\left(G^{2}\right) \leqslant \cdots \leqslant \operatorname{bind}_{\mathrm{av}}\left(G^{d}\right)=n-1$.

Proof: Since for positive integer $i, G^{i}$ is a subgraph of $G^{i+1}$, it follows from Theorem 4 that

$$
\operatorname{bind}_{\mathrm{av}}(G) \leqslant \operatorname{bind}_{\mathrm{av}}\left(G^{2}\right) \leqslant \cdots \leqslant \operatorname{bind}_{\mathrm{av}}\left(G^{d}\right) .
$$

Since $G$ is connected with diameter $d$, then $G^{d}$ is a complete graph, thus $\operatorname{bind}_{\mathrm{av}}\left(G^{d}\right)=n-1$.

\section{Join of graphs}

Definition 2 [Ref. 5] The join $G_{1}+G_{2}$ of graphs $G_{1}$ and $G_{2}$ with disjoint vertex sets $V\left(G_{1}\right)$ and $V\left(G_{2}\right)$ is the graph consists of $G_{1}, G_{2}$, and all edges joining $V\left(G_{1}\right)$ and $V\left(G_{2}\right)$.

For a join graph $G+H$, it is easy to see that

$$
\begin{aligned}
\sum_{v \in V(G+H)} \operatorname{bind}_{v}(G+H)= & \sum_{v \in V(G)} \operatorname{bind}_{v}(G+H) \\
& +\sum_{v \in V(H)} \operatorname{bind}_{v}(G+H) .
\end{aligned}
$$


Theorem 11 Let $G$ and $H$ be two connected graphs of order $m$ and $n$, respectively. Then

$$
\operatorname{bind}_{\mathrm{av}}(G+H) \geqslant \frac{m \cdot \operatorname{bind}(G)+n \cdot \operatorname{bind}(H)}{m+n} .
$$

Proof: Let $v \in V(G+H)$ and $S_{v} \in F_{v}(G+H)$. Let denote $N_{G}\left(S_{v}\right)=N\left(S_{v}\right) \cap V(G)$ and $N_{H}\left(S_{v}\right)=N\left(S_{v}\right) \cap$ $V(H)$. Since every vertex of $G$ is connected to all vertices of $H$, and vice versa, then $N\left(S_{v}\right)=N_{G}\left(S_{v}\right) \cup$ $V(H)$ or $N\left(S_{v}\right)=N_{H}\left(S_{v}\right) \cup V(G)$. Since $N\left(S_{v}\right) \neq$ $V(G+H), v \in V(G)$ implies $S_{v} \subseteq V(G)$, or $v \in V(H)$ implies $S_{v} \subseteq V(H)$.

For $v \in V(G)$, let $A_{v}^{*}$ be its local binding set in $G$. Since $S_{v} \subseteq V(G), S_{v} \in F_{v}(G+H) \cap F_{v}(G)$, thus

$$
\begin{gathered}
\frac{\left|N\left(S_{v}\right)\right|}{\left|S_{v}\right|}=\frac{\left|N_{G}\left(S_{v}\right)\right|+|V(H)|}{\left|S_{v}\right|} \geqslant \frac{\left|N_{G}\left(A_{v}^{*}\right)\right|}{\left|A_{v}^{*}\right|}+\frac{|V(H)|}{\left|S_{v}\right|}, \\
\frac{\left|N\left(S_{v}\right)\right|}{\left|S_{v}\right|}=\operatorname{bind}_{v}(G)+\frac{|V(H)|}{\left|S_{v}\right|} \geqslant \operatorname{bind}(G) .
\end{gathered}
$$

Similarly, for $v \in V(H)$,

$$
\frac{\left|N\left(S_{v}\right)\right|}{\left|S_{v}\right|} \geqslant \operatorname{bind}(H) .
$$

Hence by (1) and (2),

$$
\operatorname{bind}_{\mathrm{av}}(G+H) \geqslant \frac{m \cdot \operatorname{bind}(G)+n \cdot \operatorname{bind}(H)}{m+n} .
$$

Lemma 1 Let $G$ and $H$ be two connected graphs. If $v \in V(G)$ or $v \in V(H)$ and $S_{v}$ is local binding set of $G+H$, then $S_{v}$ is either a local binding set of $G$ or $H$.

Proof: Suppose that $v \in V(G)$. Let $N_{G}\left(S_{v}\right)=N\left(S_{v}\right) \cap$ $V(G)$. Since all vertices of $G$ is connected to all vertices of $H, N\left(S_{v}\right)=N_{G}\left(S_{v}\right) \cup V(H)$, which implies that $S_{v} \subset V(G)$. Hence $S_{v}$ is a local binding set of $G$. Similarly, if $v \in V(H)$, then $S_{v}$ is a local binding set of $H$.

Theorem 12 Let $m$ and $n$ be positive integers. Then

$$
\begin{aligned}
& \operatorname{bind}_{\mathrm{av}}\left(K_{m}+P_{n}\right) \\
& \quad= \begin{cases}m+1, & n \text { even }, \\
\frac{2 n^{2} m+2 m^{2} n+2 n^{2}-4 m^{2}-3 m n-5 n-m+3}{2 n^{2}+2 m n-4 m-4 n}, & n \text { odd. }\end{cases}
\end{aligned}
$$

Proof: Let $v \in V\left(K_{m}+P_{n}\right)$ and $S_{v} \in F_{v}\left(K_{m}+P_{n}\right)$. By Lemma 1, if $S_{v} \cap V\left(K_{m}\right) \neq \varnothing$ and $S_{v} \cap V\left(P_{n}\right) \neq \varnothing$, then $N\left(S_{v}\right)=V\left(K_{m}+P_{n}\right)$, a contradiction. Hence either $S_{v} \subseteq V\left(K_{m}\right)$ or $S_{v} \subseteq V\left(P_{n}\right)$.
If $v \in V\left(K_{m}\right)$, then $\left|S_{v}\right|=1$ otherwise $N\left(S_{v}\right)=$ $V\left(K_{m}+P_{n}\right)$. Thus $\operatorname{bind}_{v}\left(K_{m}+P_{n}\right)=(m+n-1)$ and

$$
\sum_{v \in V\left(K_{m}\right)} \operatorname{bind}_{v}\left(K_{m}+P_{n}\right)=m(m-1+n) .
$$

If $v \in V\left(P_{n}\right)$, we consider two cases.

Case 1: $n$ is even. There is a local binding set $S_{v}$ of $P_{n}$ such that $\left|S_{v}\right|=n / 2$, when $S_{v}$ contains all vertices in a maximum independent set of $P_{n}$. Then $\left|N\left(S_{v}\right)\right|=m+n / 2$ and $\operatorname{bind}_{v}\left(K_{m}+P_{n}\right)=(n+2 m) / n$. Thus

$$
\sum_{v \in V\left(P_{n}\right)} \operatorname{bind}_{v}\left(K_{m}+P_{n}\right)=n\left(\frac{n+2 m}{n}\right)=n+2 m .
$$

Hence

$$
\begin{aligned}
\operatorname{bind}_{\mathrm{av}}\left(K_{m}+P_{n}\right)= & \frac{m(m-1+n)+n+2 m}{m+n} \\
& =\frac{(m+1)(m+n)}{m+n}=m+1 .
\end{aligned}
$$

Case 2: $n$ is odd. If $v$ is in a maximum independent set of $P_{n}$, then there is a local binding set $S_{v} \subset V\left(P_{n}\right)$ such that $\left|S_{v}\right|=\beta\left(P_{n}\right)=(n+1) / 2$ and $\left|N\left(S_{v}\right)\right|=m+(n-1) / 2$. Thus $\operatorname{bind}_{v}\left(K_{m}+P_{n}\right)=$ $(2 m+n-1) /(n+1)$ for these $(n+1) / 2$ vertices $v$ in a maximum independent set of $P_{n}$.

If $v$ is not in a maximum independent set of $P_{n}$. To obtain $\operatorname{bind}_{v}\left(P_{n}\right)$, we require $S_{v}$ as large as possible that $N\left(S_{v}\right) \neq V\left(P_{n}\right)$, when $\left|S_{v}\right|=n-2$, e.g., $S_{v}=\left\{p_{3}, p_{4}, \ldots, p_{n}\right\}$, and $\left|N\left(S_{v}\right)\right|=n-1+m$. Thus $\operatorname{bind}_{v}\left(K_{m}+P_{n}\right)=(n-1+m) /(n-2)$ for these $\frac{1}{2}(n-1)$ vertices. Therefore

$$
\begin{array}{r}
\sum_{v \in V\left(P_{n}\right)} \operatorname{bind}_{v}\left(K_{m}+P_{n}\right) \\
=\frac{n+1}{2}\left(\frac{2 m+n-1}{n+1}\right)+\frac{n-1}{2}\left(\frac{n-1+m}{n-2}\right) \\
=\frac{2 n^{2}+3 m n-5 n-5 m+3}{2 n-4} .
\end{array}
$$

This implies that

$$
\begin{aligned}
& \sum_{v \in V\left(K_{m}+P_{n}\right)} \operatorname{bind}_{v}\left(K_{m}+P_{n}\right) \\
= & m(m-1+n)+\frac{2 n^{2}+3 m n-5 n-5 m+3}{2 n-4} \\
= & \frac{2 n^{2} m+2 m^{2} n+2 n^{2}-4 m^{2}-3 m n-5 n-m+3}{2 n-4} .
\end{aligned}
$$


Hence

$$
\begin{aligned}
& \operatorname{bind}_{\mathrm{av}}\left(K_{m}+P_{n}\right)=\frac{\sum_{v \in V\left(K_{m}+P_{n}\right)} \operatorname{bind}_{v}\left(K_{m}+P_{n}\right)}{m+n} \\
= & \frac{2 n^{2} m+2 m^{2} n+2 n^{2}-4 m^{2}-3 m n-5 n-m+3}{2 n^{2}+2 m n-4 m-4 n} .
\end{aligned}
$$

The proof is completed.

Theorem 13 Let $m$ and $n$ be positive integers. Then

$$
\begin{aligned}
\text { bind }_{\mathrm{av}}( & \left.K_{m}+C_{n}\right) \\
& = \begin{cases}m+1, & n \text { even, } \\
\frac{n^{2} m+m^{2} n-2 m^{2}+n^{2}-2 m n+2 m-n}{n^{2}+m n-2 m-2 n}, & n \text { odd. }\end{cases}
\end{aligned}
$$

Proof: Let $G=K_{m}+C_{n}, v \in V(G)$, and $S_{v} \in F_{v}(G)$. If $S_{v} \cap V\left(K_{m}\right) \neq \varnothing$ and $S_{v} \cap V\left(C_{n}\right) \neq \varnothing$, then $N\left(S_{v}\right)=$ $V(G)$, a contradiction. Thus $S_{v} \subseteq V\left(K_{m}\right)$ or $S_{v} \subseteq$ $V\left(C_{n}\right)$.

If $v \in V\left(K_{m}\right)$, then $\left|S_{v}\right|=1,\left|N\left(S_{v}\right)\right|=m-1+n$, and $\operatorname{bind}_{v}(G)=m-1+n$. Thus

$$
\sum_{v \in V\left(K_{m}\right)} \operatorname{bind}_{v}(G)=m(m-1+n) .
$$

If $v \in V\left(C_{n}\right)$, we consider two cases.

Case 1: $n$ is even. In this case there is a local binding set $S_{v}^{*}$ of $C_{n}$ and also $G$ such that $\left|S_{v}^{*}\right|=n / 2$ and $\left|N\left(S_{v}^{*}\right)\right|=m+n / 2$. Thus bind ${ }_{v}(G)=(n+2 m) / n$, and

$$
\sum_{v \in V\left(C_{n}\right)} \operatorname{bind}_{v}(G)=n\left(\frac{n+2 m}{n}\right)=n+2 m .
$$

Hence

$$
\operatorname{bind}_{\mathrm{av}}(G)=\frac{m(m-1+n)+(n+2 m)}{m+n}=m+1 .
$$

Case 2: $n$ is odd. In this case there is a local binding set $S_{v}^{*}$ of $C_{n}$ and also $G$ such that $\left|S_{v}^{*}\right|=n-2$, $\left|N\left(S_{v}^{*}\right)\right|=n-1+m$, and $\operatorname{bind}_{v}(G)=(n-1+m) /(n-$ 2). Thus

$$
\sum_{v \in V\left(C_{n}\right)} \operatorname{bind}_{v}(G)=n\left(\frac{n-1+m}{n-2}\right)=\frac{n^{2}-n+m n}{n-2}
$$

and

$$
\begin{gathered}
\sum_{v \in V(G)} \operatorname{bind}_{v}(G)=m(m-1+n)+\frac{n^{2}-n+m n}{n-2} \\
=\frac{n^{2} m+m^{2} n-2 m^{2}+n^{2}-2 m n+2 m-n}{n-2} .
\end{gathered}
$$

Consequently,

$$
\begin{aligned}
& \operatorname{bind}_{\mathrm{av}}(G) \\
& =\frac{n^{2} m+m^{2} n-2 m^{2}+n^{2}-2 m n+2 m-n}{(m+n)(n-2)} \\
& =\frac{n^{2} m+m^{2} n-2 m^{2}+n^{2}-2 m n+2 m-n}{n^{2}+m n-2 m-2 n} .
\end{aligned}
$$

The proof is completed.

Corollary 2 If $W_{n}$ is a wheel graph order $n+1, n \geqslant 4$, then

$$
\operatorname{bind}_{\mathrm{av}}\left(W_{n}\right)= \begin{cases}2, & n \text { even }, \\ \frac{2 n^{2}-2 n}{n^{2}-n-2}, & n \text { odd } .\end{cases}
$$

Proof: Since $W_{n}=K_{1}+C_{n}$, Theorem 13 gives bind $_{\mathrm{av}}\left(W_{n}\right)=$ bind $_{\mathrm{av}}\left(K_{1}+C_{n}\right)$ and the proof is completed.

\section{Corona of graphs}

Definition 3 [Ref. 5] The corona $G \circ H$ of two graphs $G$ and $H$ is the graph obtained by taking one copy of $G$ of order $n$ and $n$ copies $H_{i}$ of $H$, and then joining the $i$ th vertex of $G$ to every vertex of $H_{i}$.

Theorem 14 Let $G$ and $H$ be two connected graphs of order $m$ and $n$, respectively, and $A^{*}$ a binding set of $H$. Then

$$
\operatorname{bind}_{\mathrm{av}}(G \circ H) \geqslant \frac{(m-1)(n+1)+\left|N\left(A^{*}\right)\right|+1}{(m-1)(n+1)+\left|A^{*}\right|} .
$$

Proof: Let $S_{v} \in F_{v}(G \circ H)$. For $v \in V(H)$, let $A_{v}$ denote a local binding set of $H$ and note that $\left|N\left(A^{*}\right)\right| /\left|A^{*}\right| \leqslant\left|N\left(A_{v}\right)\right| /\left|A_{v}\right|$, i.e., $\operatorname{bind}(H) \leqslant$ $\operatorname{bind}_{v}(H)$. Assume $\left|A^{*}\right|=a$.

If $v \in V(G)$ or $v \in A^{*}$ then

$\left|S_{v}\right|=(m-1) n+m-1+\left|A^{*}\right|=(m-1)(n+1)+\left|A^{*}\right|$, $\left|N\left(S_{v}\right)\right|=(m-1)(n+1)+\left|N\left(A^{*}\right)\right|+1$.

If $v \in V(H)$ and $v \notin A^{*}$ then

$\left|S_{v}\right|=(m-1) n+m-1+\left|A_{v}\right|=(m-1)(n+1)+\left|A_{v}\right|$ $\left|N\left(S_{v}\right)\right|=(m-1)(n+1)+\left|N\left(A_{v}\right)\right|+1$.

It is easy to see that

$$
\begin{aligned}
& \frac{(m-1)(n+1)+\left|N\left(A^{*}\right)\right|+1}{(m-1)(n+1)+\left|A^{*}\right|} \\
& \leqslant \frac{(m-1)(n+1)+\left|N\left(A_{v}\right)\right|+1}{(m-1)(n+1)+\left|A_{v}\right|} .
\end{aligned}
$$


So we have

$$
\begin{gathered}
\sum_{v \in V(G \circ H)} \operatorname{bind}_{v}(G \circ H) \\
=((m-1)(n+1)+a) \frac{(m-1)(n+1)+\left|N\left(A^{*}\right)\right|+1}{(m-1)(n+1)+\left|A^{*}\right|} \\
\quad+(n-a) \frac{(m-1)(n+1)+\left|N\left(A_{v}\right)\right|+1}{(m-1)(n+1)+\left|A_{v}\right|} \\
\geqslant(m n+m) \times \frac{(m-1)(n+1)+\left|N\left(A^{*}\right)\right|+1}{(m-1)(n+1)+\left|A^{*}\right|} .
\end{gathered}
$$

Thus

$$
\begin{aligned}
\operatorname{bind}_{\mathrm{av}}(G \circ H) & =\frac{1}{m n+m} \sum_{v \in V(G \circ H)} \operatorname{bind}_{v}(G \circ H) \\
& \geqslant \frac{(m-1)(n+1)+\left|N\left(A^{*}\right)\right|+1}{(m-1)(n+1)+\left|A^{*}\right|} .
\end{aligned}
$$

The lower bound in Theorem 14 is the best possible for some graphs as in Corollary 3 and 4 .

Corollary 3 Let $G$ be a connected graph of order $m$. Then

$$
\operatorname{bind}_{\mathrm{av}}\left(G \circ K_{n}\right)=\frac{(m-1)(n+1)+n}{(m-1)(n+1)+1} .
$$

Corollary 4 Let $G$ be a connected graph of order $m$. If $n$ is even, then

$$
\begin{aligned}
& \operatorname{bind}_{\mathrm{av}}\left(G \circ P_{n}\right)=\frac{(m-1)(n+1)+\frac{n}{2}+1}{(m-1)(n+1)+\frac{n}{2}}, \\
& \operatorname{bind}_{\mathrm{av}}\left(G \circ C_{n}\right)=\frac{(m-1)(n+1)+\frac{n}{2}+1}{(m-1)(n+1)+\frac{n}{2}} .
\end{aligned}
$$

\section{Cartesian product of graphs}

Definition 4 [Ref. 5] The Cartesian product $G_{1} \times G_{2}$ of graphs $G_{1}$ and $G_{2}$ has the vertex set $V\left(G_{1}\right) \times$ $V\left(G_{2}\right)$, where $\left(u_{1}, u_{2}\right)$ is adjacent to $\left(v_{1}, v_{2}\right)$ if either $u_{1}=v_{1}$ and $u_{2}$ is adjacent to $v_{2}$ or $u_{2}=v_{2}$ and $u_{1}$ is adjacent to $v_{1}$.

Theorem 15 (Ref. 11) For graphs $G$ and $H$, if $\operatorname{bind}(G) \geqslant 1$, then $\operatorname{bind}(G \times H) \geqslant 1$.

Theorem 16 For graphs $G$ and $H$, if $\operatorname{bind}(G) \geqslant 1$, then $\operatorname{bind}_{\mathrm{av}}(G \times H) \geqslant 1$.

Proof: By Theorem 15, if $\operatorname{bind}(G) \geqslant 1$, then $\operatorname{bind}(G \times H) \geqslant 1$. It follows from Theorem 5 that $\operatorname{bind}_{\mathrm{av}}(G \times H) \geqslant \operatorname{bind}(G \times H) \geqslant 1$.

The lower bound in Theorem 16 is the best possible for come graphs as in Corollary 5 .
Corollary 5 Let $m$ and $n$ be even integers. Then

(i) $\operatorname{bind}_{\mathrm{av}}\left(P_{m} \times P_{n}\right)=1$.

(ii) $\operatorname{bind}_{\mathrm{av}}\left(P_{m} \times C_{n}\right)=1$.

(iii) $\operatorname{bind}_{\mathrm{av}}\left(C_{m} \times C_{n}\right)=1$.

\section{CONCLUSIONS}

In this study, a new graph theoretical parameter, namely, the average binding number, as the average of the local binding number of every vertex of a graph, has been presented for the network vulnerability. Additionally, the stability of popular interconnection networks has been studied and the average binding numbers have been computed. The average binding number gives the meaning that, if $\operatorname{bind}_{\mathrm{av}}(G)$ is large, then the vertices of $G$ are well bound together in the sense that $G$ has a lot of fairly well distributed edges.

\section{REFERENCES}

1. Aslan E (2014) The average lower connectivity of graphs. J Appl Math 2014, 807834.

2. Aslan E, Kilinc D, Yucalar F, Borandag E (2016) The average scattering number of graphs. RAIRO - Theor Inf Appl 50, 263-72.

3. Aytac V (2012) Average lower domination number in graphs. CR Acad Bulg Sci 65, 1665-74.

4. Chartrand G, Lesniak I (1996) Graphs and Digraphs, 3rd edn, Chapman Hall, Orange, CA.

5. Harary F (1994) Graph Theory, Addison-Wesley, NY.

6. Henning MA, Oellermann OR (2004) The average connectivity of a digraph. Discrete Appl Math 140, 143-53.

7. Kane VG, Mohanty SP, Hales HS (1981) Product graphs and binding numbers. Ars Combinatoria 11, 201-24.

8. Kane VG, Mohanty SP (1981) Binding number, cycles and complete graphs. In: Combinatorics and Graph Theory (Calcutta, 1980), Lecture Notes in Maths 885 , Springer, Berlin, pp 290-6.

9. Linzhung L, Zhongfu Z, Jianfang W (2001) On the edge-binding number of some plane graphs. Acta Math Appl Sin 17, 443-8.

10. Turaci T (2016) On the average lower bondage number of a graph. RAIRO-Oper Res 50, 1003-12.

11. Wang J, Tian S, Liu J (1984) The binding number of product graphs. In: Koh KM, Yap HP (eds) Graph Theory Singapore 1983, Lecture Notes in Mathematics 1073, Springer, Berlin, Heidelberg, pp 119-28.

12. Wang J, Tian S, Liu J (1985) The binding number of lexicographic products of graphs. In: Alavi Y, Chartrand G, Lick DR, Wall CE, Lesniak L (eds) Graph Theory with Applications to Algorithms and Computer Science, John Wiley \& Sons, New York, 761-76.

13. Woodall DR (1973) The binding number of a graph and its Anderson number. $J$ Comb Theory B 15, $225-55$. 